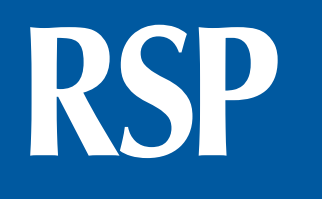

http://www.rsp.fsp.usp.br/

Revista de Saúde Pública

\title{
Pattern evolution of antidepressants and benzodiazepines use in a cohort
}

\author{
Geisy de Carvalho Alcantara' (iD, Evandro Silva Freire Coutinho" (iD, Eduardo Faerstein" iD \\ ' Universidade do Estado do Rio de Janeiro. Instituto de Medicina Social. Programa de Pós-Graduação em Saúde \\ Coletiva. Rio de Janeiro, RJ, Brasil \\ " Universidade do Estado do Rio de Janeiro. Instituto de Medicina Social. Departamento de Epidemiologia. Rio \\ de Janeiro, RJ, Brasil
}

\begin{abstract}
OBJECTIVE: In recent decades there has been an increase in the use of antidepressants (AD) and a decrease in the use of benzodiazepines (BDZ). Prevalence, cumulative incidence, and factors associated with the incidence of AD and BDZ use in a Brazilian population were estimated in this article.
\end{abstract}

METHODS: Data were collected with a self-administered questionnaire in a cohort of employees from a university in Rio de Janeiro. The prevalence of the use of AD and BDZ was calculated for 1999 (4,030), 2001 (3,574), 2006-07 (3,058), and 2012 (2,933). The cumulative incidences of the use of AD and BDZ between 1999 and 2007 were estimated by the Poisson models with robust variance estimates.

RESULTS: In 1999, the prevalence of the use of AD and BDZ were 1.4\% (95\%CI: 1.1-1.8) and 4.7\% (95\%CI: 4.1-5.4), respectively; in 2012, they were 5.4\% (95\%CI: 5.5-6.2) and 6.8\% (95\%CI: 6.0-7.8). The incidence of use, between 1999 and 2007, was 4.9\% (95\%CI: 4.2-5.7) for AD and 8.3\% (95\%CI: 7.3-9.3) for BDZ. The incidences of AD and BDZ use were higher among women and participants with a positive General Health Questionnaire.

CONCLUSION: In this population, the increase in the use of AD was not accompanied by a decrease in the use of BDZ, showing the prescriptions for psychotropic medication do not follow the currently recommended guidelines for treatment of common mental health disorders.

\author{
Correspondence: \\ Geisy de Carvalho Alcantara \\ Rua São Francisco Xavier, 524 - \\ Maracanã, Bloco D, $7^{\circ}$ andar - UERJ \\ E-mail: geisycarvalho2011@gmail. \\ com
}

Received: Jun 30, 2019

Approved: Aug 29, 2019

How to cite: Alcantara GC, Coutinho ESF, Faerstein E. Pattern evolution of antidepressants and benzodiazepines use in a cohort. Rev Saude Publica. 2020;54:40.

Copyright: This is an open-access article distributed under the terms of the Creative Commons Attribution License, which permits unrestricted use, distribution, and reproduction in any medium, provided that the original author and source are credited.
DESCRIPTORS: Benzodiazepines, administration \& dosage. Antidepressive Agents, administration \& dosage. Drug Utilization, trends. Cohort Studies. 


\section{INTRODUCTION}

Antidepressants (AD) and benzodiazepines (BDZ) are the most used psychotropic medications. The first AD was introduced in the 1950s and presented important side effects and contraindications, while BDZ showed fewer side effects and were considered to be safer, resulting in their more frequent prescription by psychiatrists and non-psychiatrists alike. With the introduction of fluoxetine, a selective serotonin reuptake inhibitors (SSRI) in the 1980s with fewer side effects, the AD use increased more than the observed for BDZ ${ }^{1,2}$. International guidelines recommend the use of BDZ should be restricted to patients with major depression disorders associated with anxiety and/or insomnia, and only if the $\mathrm{AD}$ did not provide an adequate treatment ${ }^{2,3}$.

In 1988, in the United States (US), 9.7\% of patients with a depression diagnosis received a prescription for the SSRI fluoxetine. This proportion gradually rose to $21 \%$ (1989), $46 \%$ (1993), and 69\% (2001). In parallel, there was a decrease in the use of BDZ for depression treatment: in 1987, 21\% of individuals used BDZ for this purpose, but in 2001 the proportion was only $7.5 \%^{1}$.

There is significant variation in the prevalence of use of $\mathrm{AD}$ and $\mathrm{BDZ}$ in the general population among countries, for example $2.9 \%$ (AD) and 3.8\% (BDZ) in Chile in 1996-984; 4.7\% (AD) and $11.4 \%$ (BDZ) in Spain in 2001-025 ; and 7.2\% (AD) and 12.3\% (BDZ) in Belgium in 2001-02 . A study conducted in 2000 in six European countries (Belgium, France, Germany, Italy, Netherlands and Spain), estimated 3.7\% annual prevalence for AD and 9.8\% for BDZ7.

In Brazil, a study in 1988 among 1,459 residents in Rio de Janeiro found a 5.2\% prevalence for the use of psychotropic medicine in the previous month, $85 \%$ being BDZ ${ }^{8}$. Around 20 years later, in another study in $\mathrm{Rio}^{9}$, the most used type of psychotropic medicines in the previous month was the AD, with $2.8 \%$, and the prevalence of BDZ use was $1.6 \%$. Several studies have been conducted in São Paulo. In 1990, among 1,742 individuals, only five had used $\mathrm{AD}$ in the previous 12 months, compared with the 140 that had used BDZ, a prevalence of $8 \%^{10}$. Later (2007), one study estimated similar prevalence of BDZ (3.7\%) and AD (3.5\%) use in the previous 12 months $^{11}$, and in another one the prevalence of AD use in the previous 30 days was $3.1 \%$, and BDZ use was $2.7 \%^{12}$. Studies point to greater use of psychotropic medicine by women, older adults, those with a higher income and education (especially in the AD case), the unemployed, and people who are separated or divorced ${ }^{2,9,11-14}$. Some findings are inconsistent as some studies report, for example, a higher prevalence among less educated individuals ${ }^{2,8}$.

The objective of this study is to investigate the prevalence and cumulative incidence of $\mathrm{AD}$ and BDZ among Brazilian adults and factors associated with the use of these medicines. This study hypothesizes that the use of AD has been increasing since the year 2000 while the use of BDZ has been decreasing.

\section{METHODS}

\section{Study design}

A concurrent cohort study with data from the Pró-Saúde Study, a longitudinal study of university civil servants in Rio de Janeiro ${ }^{15}$.

The wave 1 of the study (baseline) occurred in 1999, with 4,030 participants (91\% of those eligible); wave 2 occurred in 2001-2 (3,574 individuals, $83 \%$ of those eligible); wave 3 occurred in 2006-7, with 3,058 individuals (76\% of those eligible); wave 4 occurred in 2012, with $2,933$ individuals ( $58 \%$ of those eligible) $)^{15}$.

The ethics committee of the Universidade Estadual do Rio de Janeiro approved the research protocol; all subjects signed an informed consent form. 


\section{Variables}

The variables used in the study were obtained with a self-administered questionnaire applied during wave 1 (1999):

- Sociodemographic (sex, age, marital state, self-reported race/skin color, education level, and equivalent income compared with minimum wage at the time).

- Use of alcohol. This variable was assessed by three questions: i. "Did you consume any type of alcoholic drink in the last two weeks?" ii. "How many days did you consume any type of alcoholic drink in the last two weeks?" iii. "How many doses did you consume in the days you drank in the last two weeks?"

- Self-reported health assessment (general health condition and current health condition).

- Sleep problems (difficulty in initiating sleep and waking up frequently at night).

- Tiredness for no apparent reason.

- Mental health was assessed with the Brazilian version of the General Health Questionnaire - GHQ-12 (validated by a structured psychiatric interview) ${ }^{16}$ during waves 1,2 , and 3 of the study. The GHQ-12 scores were dichotomized, using scores from 3 or more as indicative of common mental disorder (CMD).

- The use of psychotropic drugs was assessed with the construction of a dichotomous variable (no/yes) based on a question referring to medicine use: "During the past two weeks, have you used any medicine? If the answer is yes, which medicine have you used in the past two weeks?". During waves 3 and 4, the information about medicine use regarded the previous seven days.

The participants were classified as AD or BDZ users if reported (i) the name of the substance or (ii) the pharmacological class. If the name of the medicine was not reported, the Anatomical Therapeutic Chemical (ATC) classification was used ${ }^{17}$.

\section{Analysis}

A descriptive analysis of the study population was performed with measures estimations of central tendency and dispersion for the continuous variables and frequency distribution for the categorical variables. Prevalence and $95 \%$ confidence interval (CI) were estimated for the use of AD and BDZ.

Then a fixed cohort with individuals who did not use AD or BDZ during wave 1 was assembled and observed until wave 3, thus providing eight years of follow-up. Cumulative incidences of $\mathrm{AD}$ and $\mathrm{BDZ}$ were estimated in both phases following wave 1 of the study. Wave 4 was excluded from the follow-up analysis due to many study participants who quit the follow-up. In an exploratory data analysis, incidences were estimated and stratified by sociodemographic, general, and mental health variables. The initial choice of variables among the ones collected in the original study considered the relationship of the variables with the use of AD and BDZ present in the literature. The associations were considered statistically significant if $p$-value $\leq 0.05$, and of borderline statistical significance if between 0.06 and 0.10 .

To estimate the cumulative incidence ratio (relative risk) the Poisson regression models with robust variance estimates were adjusted, having as a dependent variable the incidence of $\mathrm{AD}$ and $\mathrm{BDZ}$ use. Initially, the models were adjusted with only one independent variable at a time. Variables associated with $p$-values $\leq 0.20$ were selected for subsequent assessment in the multivariate models, with their inclusion one by one; those associated with $\mathrm{p}$-values $>0.10$ were kept in the model.

The analysis was performed using the statistical software Stata version 12. 


\section{RESULTS}

\section{Population characteristics during wave 1 (baseline)}

The overall sample in wave 1 contained 4,030 individuals. Most of them were female (55\%), ranging from 35 to 44 years of age (43\%). Most participants were married/cohabiting (60\%), self-reported as being white (52\%), who completed higher education (27\%), and worked at the university hospital (49\%). Two-thirds of the individuals had an income greater than six times the minimum wage. More than half the individuals (56\%) reported having private health insurance (Table 1).

Almost half the participants classified their health as good, and two-thirds considered their current health condition to be the same as 12 months before. Mental health assessment with the GHQ-12 showed 31\% met the criteria for CMD. Two-thirds of the individuals said they never or rarely had trouble falling asleep. Also they never or rarely woke up during the sleep cycle. More than half the participants (56\%) never or rarely declared tiredness for no reason (Table 1).

\section{Prevalence of antidepressant and benzodiazepine use}

The prevalence of $\mathrm{AD}$ and $\mathrm{BDZ}$ use was higher among women. The greatest difference was in $\mathrm{AD}$ use, with women presenting a prevalence of approximately four times higher than men (Table 1).

Overall, the prevalence of $\mathrm{AD}$ use increased with age. As for the BDZ, there was an increase in usage by people between 45 and 54 years old. The prevalence of both AD and BDZ use was lower in married individuals, and for BDZ it was also lower in the single group (Table 1).

The prevalence of $\mathrm{AD}$ and $\mathrm{BDZ}$ use lacked distinctions by race/color, education level, income, and possession of private health insurance. Regarding the workplace, the only difference in prevalence was in BDZ use, which was 35\% higher among participants at the university hospital (Table 1).

Increased use of AD and BDZ was noticed when the individual had a worse health perception. Among individuals with $\mathrm{CMD}$, the prevalence of $\mathrm{AD}$ and $\mathrm{BDZ}$ use was close to four times higher. There was an increasing gradient of AD and BDZ use with the intensity of sleep complaints or with tiredness for no apparent reason (Table 1).

In 1999, the prevalence of AD use in the 15 previous days was reported by $1.4 \%$ of participants (95\%CI: 1.1-1.8); in 2012, even over a smaller span (the previous seven days), the prevalence was 5.4\% (95\%CI: 4.6-6.2). As for the prevalence of BDZ, there was a progressive, although discreet, increase in use with an apparent tendency to stability: being 4.7\% (95\%CI: 4.1-5.4) in 1999 and 6.8\% (95\%CI: 6.0-7.8) in 2012 (Figure 1).

In 1999, 58 individuals reported using AD in the previous two weeks. Tricyclic antidepressants (TCA), which represented in $199960.3 \%$ of the used AD, decreased to $15.2 \%$ in 2012. In contrast, SSRI boosted the increase in AD use, from 29.3\% in 1999 to 67.6\% in 2012, being primarily responsible for this increase. In parallel, there was a significant decrease in the use of bromazepam, from $43.7 \%$ in 1999 to $12.0 \%$ in 2012 (Table 2).

\section{Incidence of antidepressant and benzodiazepine use}

There were increases in $\mathrm{AD}$ and $\mathrm{BDZ}$ use throughout the study period. The incidence of $\mathrm{AD}$ in 2001 was $1.5 \%$, and $3.4 \%$ for BDZ. For the years of 2006-7, the cumulative incidence of eight years after wave 1 (baseline) were $3.6 \%$ for $\mathrm{AD}$ and $5.0 \%$ for BDZ.

The incident cases in both waves after the baseline totaled 150 individuals (4.9\%) for AD and $243(8.3 \%)$ for BDZ. The incidence among women was 3.4 times higher for AD and twice as high for BDZ than among men. Individuals aged 55 years or older used AD the least (1.7\%); the opposite was found in the incidence of BDZ (13.3\%). Widowers were those who most started using $\mathrm{AD}(7.4 \%)$ and $\mathrm{BDZ}(22.2 \%)$ in the follow-up period. (Table 3 ). 
Table 1. Sociodemographic characteristics, self-reported health conditions, and prevalence of antidepressants and benzodiazepines use at the baseline (1999). Pró-Saúde Study, Brazil.

\begin{tabular}{|c|c|c|c|c|c|}
\hline & $\begin{array}{c}\text { Baseline }(4,030) \\
\text { N (\%) }\end{array}$ & $\begin{array}{c}\text { Prevalence of AD \% } \\
(95 \% \mathrm{CI})\end{array}$ & p-value & $\begin{array}{c}\text { Prevalence of BDZ \% } \\
(95 \% \mathrm{Cl})\end{array}$ & p-value \\
\hline Total & & $1.4(1.1-1.8)$ & & $4.7(4.0-5.4)$ & \\
\hline \multicolumn{6}{|l|}{ Sex } \\
\hline Men & $1,792(44.5)$ & $0.5(0.2-0.9)$ & \multirow{2}{*}{$<0.001$} & $2.8(2.1-3.6)$ & \multirow{2}{*}{$<0.001$} \\
\hline Women & $2,238(55.5)$ & $2.1(1.5-2.7)$ & & $6.2(5.2-7.2)$ & \\
\hline \multicolumn{6}{|l|}{ Age } \\
\hline $22-34$ & $1,124(27.9)$ & $1.3(0.7-2.0)$ & \multirow{4}{*}{0.922} & $2.2(1.4-3.1)$ & \multirow{4}{*}{$<0.001$} \\
\hline $35-44$ & $1,740(43.1)$ & $1.4(0.8-1.9)$ & & $5.0(4.0-6.1)$ & \\
\hline $45-54$ & $885(22.0)$ & $1.6(0.7-2.4)$ & & $7.0(5.3-8.7)$ & \\
\hline 55 or more & $281(7.0)$ & $1.8(0.2-3.3)$ & & $5.3(2.7-8.0)$ & \\
\hline \multicolumn{6}{|l|}{ Marital status } \\
\hline Married or living together & 2,397 (59.5) & $1.0(0.6-1.4)$ & \multirow{5}{*}{0.06} & $4.1(3.3-4.9)$ & \multirow{5}{*}{$<0.001$} \\
\hline Separated or divorced & $611(15.0)$ & $2.1(1.0-3.3)$ & & $8.3(6.1-10.5)$ & \\
\hline Widower & $116(3.0)$ & $2.6(0.0-5.5)$ & & $6.9(2.3-11.5)$ & \\
\hline Single & $805(20.0)$ & $2.0(1.0-2.9)$ & & $3.5(2.2-4.7)$ & \\
\hline No information & $101(2.5)$ & - & & - & \\
\hline \multicolumn{6}{|l|}{ Race } \\
\hline Black & $626(15.5)$ & $1.4(0.5-2.4)$ & \multirow{6}{*}{0.811} & $4.9(3.2-6.6)$ & \multirow{6}{*}{0.576} \\
\hline Brown & $1,171(29.1)$ & $1.4(0.8-2.1)$ & & $4.4(3.2-5.6)$ & \\
\hline White & $2,082(51.7)$ & $1.5(1.0-2.1)$ & & $5.0(4.0-5.9)$ & \\
\hline Asian & $60(1.5)$ & - & & $3.3(-1.2-7.9)$ & \\
\hline Indigenous & $41(1.0)$ & - & & - & \\
\hline No information & $50(1.2)$ & - & & - & \\
\hline \multicolumn{6}{|l|}{ Education level } \\
\hline Some elementary school & $276(6.8)$ & $1.1(-0.1-2.3)$ & \multirow{8}{*}{0.951} & $5.1(2.5-7.7)$ & \multirow{8}{*}{0.609} \\
\hline Elementary school & $305(7.6)$ & $1.6(0.2-3.1)$ & & $3.9(1.7-6.1)$ & \\
\hline Some high school & $365(9.0)$ & $1.4(0.2-2.6)$ & & $6.6(4.0-9.1)$ & \\
\hline High school & $870(21.6)$ & $1.6(0.8-2.4)$ & & $4.8(3.4-6.2)$ & \\
\hline Some college & $560(14.0)$ & $1.2(0.3-2.2)$ & & $3.7(2.2-5.3)$ & \\
\hline College & $1,084(27.0)$ & $1.3(0.6-2.0)$ & & $4.7(3.4-6.0)$ & \\
\hline Graduate & $523(13.0)$ & $1.9(0.7-3.1)$ & & $4.6(2.8-6.4)$ & \\
\hline No information & $47(1.0)$ & - & & - & \\
\hline \multicolumn{6}{|l|}{ Workplace } \\
\hline UERJ' Campus & $2,053(51.0)$ & $1.2(0.7-1.7)$ & \multirow{2}{*}{0.229} & $4.0(3.2-4.9)$ & \multirow{2}{*}{0.04} \\
\hline HUPE² Hospital & $1,977(49.0)$ & $1.7(1.1-2.2)$ & & $5.4(4.4-6.4)$ & \\
\hline \multicolumn{6}{|c|}{ Income (equal to number of minimum wages) } \\
\hline Up to 3 & $329(8.7)$ & $1.2(0.02-2.4)$ & \multirow{3}{*}{0.6} & $5.7(3.2-8.3)$ & \\
\hline 3 to 6 & $967(25.5)$ & $1.1(0.5-1.8)$ & & $4.4(3.1-5.7)$ & 0.619 \\
\hline$>6$ & $2,490(65.8)$ & $1.6(1.1-2.0)$ & & $4.7(3.9-5.6)$ & \\
\hline Health insurance users & & & & & \\
\hline Yes & $2,266(56.2)$ & $1.5(0.9-2.0)$ & & $4.9(4.0-5.9)$ & \\
\hline No & $1,739(43.1)$ & $1.3(0.8-1.8)$ & 0.638 & $4.4(3.4-5.4)$ & 0.446 \\
\hline No information & $25(0.6)$ & - & & - & \\
\hline Alcohol consumption & & & & & \\
\hline Yes & $2,148(53.3)$ & $1.1(0.6-1.5)$ & 003 & $3.9(3.1-4.8)$ & 01 \\
\hline No & $1,751(43.5)$ & $1.9(1.2-2.5)$ & 0.03 & $5.7(4.6-6.8)$ & 0.01 \\
\hline No information & $131(3.2)$ & - & & - & \\
\hline Frequency of alcohol consum & & & & & \\
\hline Every day & $84(2.1)$ & $1.2(0.0-3.5)$ & & $5.9(0.8-11.0)$ & \\
\hline 10 to 13 days & $77(1.9)$ & - & & $2.6(0.0-6.2)$ & \\
\hline 6 to 9 days & $172(4.3)$ & $0.6(0.0-1.7)$ & 0.73 & $3.5(0.7-6.2)$ & 0.76 \\
\hline 2 to 5 days & $1.098(27.2)$ & $1.0(0.4-1.6)$ & & $4.2(3.0-5.4)$ & \\
\hline 1 single day & 708 (17.6) & $1.4(0.5-2.3)$ & & $3.5(2.2-4.9)$ & \\
\hline
\end{tabular}


Table 1. Sociodemographic characteristics, self-reported health conditions, and prevalence of antidepressants and benzodiazepines use at the baseline (1999). Pró-Saúde Study, Brazil. (Continuation)

\begin{tabular}{|c|c|c|c|c|c|}
\hline & $\begin{array}{c}\text { Baseline }(4,030) \\
N(\%)\end{array}$ & $\begin{array}{c}\text { Prevalence of AD \% } \\
(95 \% \mathrm{CI})\end{array}$ & p-value & $\begin{array}{c}\text { Prevalence of BDZ \% } \\
(95 \% \mathrm{Cl})\end{array}$ & p-value \\
\hline \multicolumn{6}{|c|}{ Amount of alcohol consumed per day ( 2 weeks) } \\
\hline 1 standard unit & $631(15.7)$ & $1.1(0.3-1.9)$ & \multirow{5}{*}{0.98} & $3.2(1.8-4.5)$ & \multirow{5}{*}{0.3} \\
\hline 2 to 4 standard unit & $902(22.4)$ & $1.1(0.4-1.8)$ & & $4.1(2.8-5.4)$ & \\
\hline 5 to 7 standard unit & $311(7.7)$ & $1.0(0.0-2.0)$ & & $3.2(1.2-5.2)$ & \\
\hline 8 a 10 standard unit & $141(3.5)$ & $1.4(0.0-3.4)$ & & $5.0(1.4-8.6)$ & \\
\hline More than 10 standard unit & $131(3.2)$ & $0.8(0.0-2.3)$ & & $6.9(2.5-11.2)$ & \\
\hline \multicolumn{6}{|l|}{ Health condition } \\
\hline Very good & $1,132(28.1)$ & $0.4(0.05-0.8)$ & \multirow{5}{*}{$<0.001$} & $1.8(1.0-2.5)$ & \multirow{5}{*}{$<0.001$} \\
\hline Good & $2,131(52.9)$ & $1.2(0.7-1.6)$ & & $4.3(3.4-5.1)$ & \\
\hline Regular & $683(16.9)$ & $3.2(2.0-4.5)$ & & $9.9(7.7-12.2)$ & \\
\hline Bad & $63(1.6)$ & $9.5(2.2-16.8)$ & & $14.3(5.6-23.0)$ & \\
\hline No information & $21(0.5)$ & - & & - & \\
\hline \multicolumn{6}{|l|}{ Current health condition } \\
\hline Better than 12 months ago & $784(19.5)$ & $2.4(1.3-3.5)$ & \multirow{3}{*}{$<0.001$} & $5.3(3.8-6.9)$ & \multirow{4}{*}{$<0.001$} \\
\hline Same as 12 months ago & $2,721(67.5)$ & $0.9(0.5-1.2)$ & & $3.3(2.6-4.0)$ & \\
\hline Worse than 12 months ago & $501(12.4)$ & $3.0(1.5-4.5)$ & & $11.4(8.6-14.2)$ & \\
\hline No information & $24(0.6)$ & - & & - & \\
\hline \multicolumn{6}{|l|}{ GHQ-12 (positive $\geq 3$ ) } \\
\hline Negative & $2,740(68.0)$ & $0.7(0.4-1.0)$ & \multirow{3}{*}{$<0.001$} & $2.3(1.8-2.9)$ & \multirow{3}{*}{$<0.001$} \\
\hline Positive & $1,233(30.6)$ & $3.1(2.1-4.0)$ & & $10.1(8.4-11.8)$ & \\
\hline No information & $57(1.4)$ & - & & - & \\
\hline \multicolumn{6}{|l|}{ Difficulty falling asleep } \\
\hline Always & $178(4.4)$ & $5.0(1.8-8.3)$ & \multirow{6}{*}{$<0.001$} & $23.6(17.3-29.8)$ & \multirow{6}{*}{$<0.001$} \\
\hline Almost Always & $297(7.4)$ & $5.5(2.5-7.5)$ & & $14.5(10.5-18.5)$ & \\
\hline Sometimes & $933(23.1)$ & $1.7(0.9-2.5)$ & & $5.5(4.0-7.0)$ & \\
\hline Rarely & $887(22.0)$ & $0.8(0.2-1.4)$ & & $2.7(1.6-3.8)$ & \\
\hline Never & $1,699(42.2)$ & $0.6(0.2-1.0)$ & & $1.5(0.9-2.1)$ & \\
\hline No information & $36(0.9)$ & - & & - & \\
\hline \multicolumn{6}{|l|}{ Wake up during sleep cycle } \\
\hline Always & $139(3.5)$ & $4.3(0.9-7.7)$ & \multirow{6}{*}{$<0.001$} & $23.7(16.6-30.8)$ & \multirow{6}{*}{$<0.001$} \\
\hline Almost always & $320(7.9)$ & $3.7(1.7-5.8)$ & & $11.2(7.8-14.7)$ & \\
\hline Sometimes & $902(22.4)$ & $2.0(1.1-2.9)$ & & $6.8(5.1-8.4)$ & \\
\hline Rarely & $915(22.7)$ & $1.5(0.7-2.3)$ & & $3.7(2.5-4.9)$ & \\
\hline Never & $1,723(42.7)$ & $0.5(0.1-0.8)$ & & $1.4(0.8-1.9)$ & \\
\hline No information & $31(0.8)$ & - & & - & \\
\hline \multicolumn{6}{|l|}{ Tiredness for no apparent reason } \\
\hline Always & $191(4.7)$ & $4.2(1.3-7.0)$ & \multirow{5}{*}{$<0.001$} & $16.2(11.0-21.5)$ & \multirow{6}{*}{$<0.001$} \\
\hline Almost always & $423(10.5)$ & $4.5(2.5-6.5)$ & & $10.2(7.3-13.0)$ & \\
\hline Sometimes & $1,107(27.5)$ & $1.5(0.8-2.3)$ & & $5.5(4.2-6.8)$ & \\
\hline Rarely & $765(19.0)$ & $1.0(0.3-1.8)$ & & $2.9(1.7-4.1)$ & \\
\hline Never & $1,504(37.3)$ & $0.3(0.0-0.6)$ & & $1.9(1.2-2.6)$ & \\
\hline No information & $40(1.0)$ & - & & - & \\
\hline
\end{tabular}

${ }^{1}$ Universidade Estadual do Rio de Janeiro

${ }^{2}$ Hospital University Pedro Ernesto 


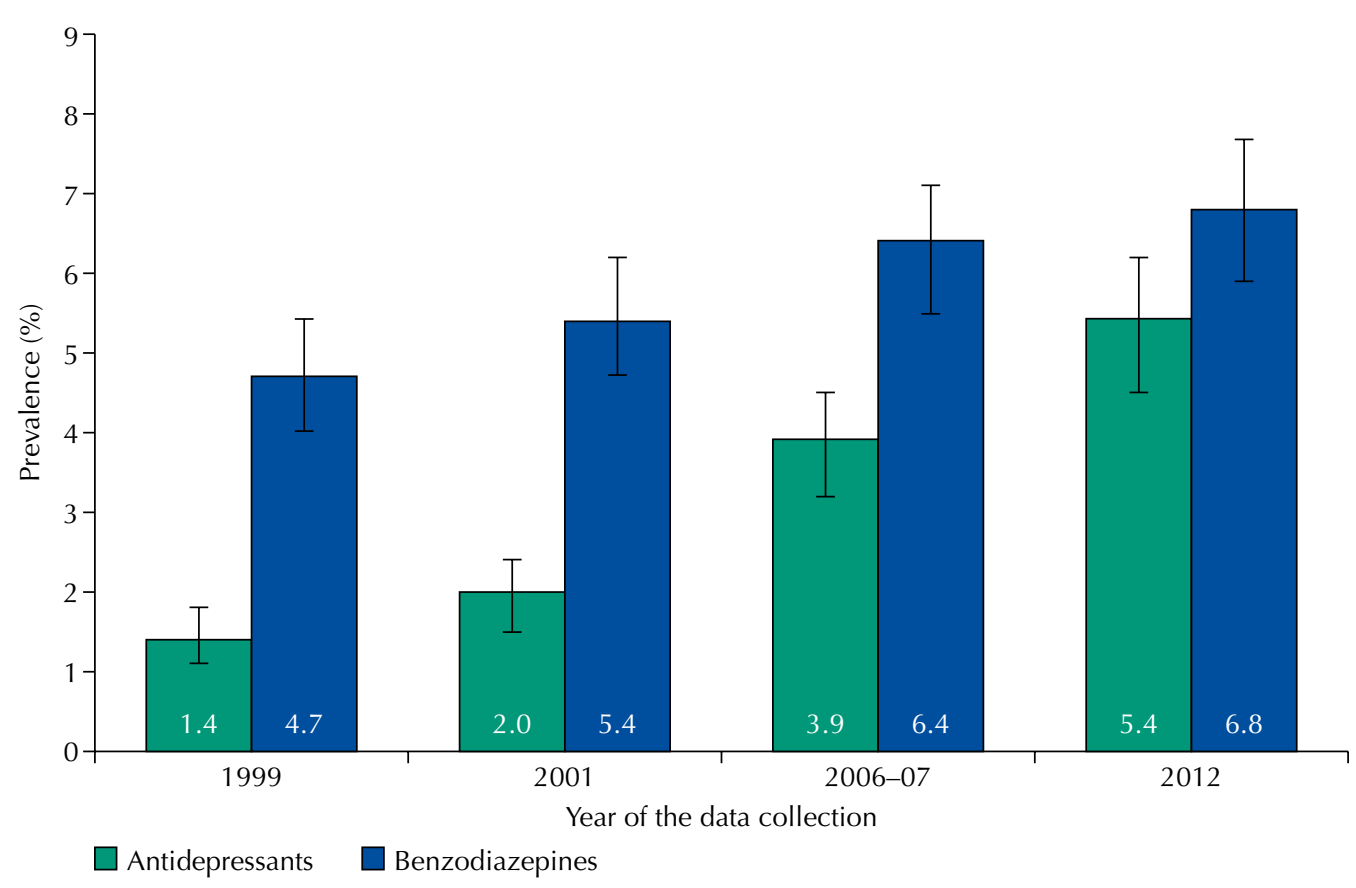

1999 and 2001: two-weeks prevalence; 2006-2007 and 2012: one-week prevalence

Figure 1. Prevalence of antidepressants and benzodiazepines in the four cohort waves (1999, 2001, 2006-07, 2012). Pró-Saúde Study, Brazil.

There was greater $\mathrm{AD}$ use among better educated individuals, whereas $\mathrm{BDZ}$ use was more uniform among the educational categories. The incidences of AD and BDZ use were $39 \%$ higher among individuals who worked at the university hospital. The incidence of AD and BDZ use was higher among those who reported having poor or regular health. Individuals who declared their health to be worse than it was 12 months before were shown to have higher incidence, although in BDZ cases this was also observed for those who declared their health to be better than before. The incidence of AD and BDZ use practically doubled among individuals with CMD. Most participants who started to use AD and BDZ after wave 1 reported sleep complaints and tiredness for no reason (Table 3).

The incidence of AD use among BDZ users and non-users was investigated at the baseline and was three times higher among individuals who used BDZ (12.2\%) compared with those who did not (4.6\%).

\section{Multivariate models}

\section{Antidepressants}

Women presented a cumulative incidence of AD use almost two and a half times higher than men in the studied period. Using the younger age group (22-34 years) as a reference, there was an increase of about $50 \%$ in the incidence in the 45-54 years age group, and a decrease by half in the group aged over 55 , although the p-value reached a borderline level of significance $(\mathrm{p}=0.06)$. The incidence of AD use for individuals with higher education, even if incomplete, was more than two times that for individuals without any higher education. Individuals suspected to have CMD (GHQ $\geq 3$ ) had an AD use $34 \%$ higher than those with no disorder, but with a borderline p-value (0.08). The complaint of tiredness presented a dose-response relationship with the use of these psychotropic medicines (Table 4).

\section{Benzodiazepines}

Women presented a cumulative incidence 58\% higher than men in the studied period. Individuals self-classified as brown, black, and indigenous individuals were shown to have higher risk of being a BDZ incident user. The incidence of these medicines among widowers was 
Table 2. Distribution of antidepressants and benzodiazepines users according to class and medications (1999, 2001, 2006-2007, 2012). Pró-Saúde Study, Brazil.

\begin{tabular}{|c|c|c|c|c|}
\hline Classes/medications & $\begin{array}{c}\text { Phase } 1 \\
\mathrm{~N}(\%)\end{array}$ & $\begin{array}{c}\text { Phase } 2 \\
\text { N (\%) }\end{array}$ & $\begin{array}{c}\text { Phase } 3 \\
\text { N (\%) } \\
\end{array}$ & $\begin{array}{c}\text { Phase } 4 \\
\mathrm{~N}(\%)\end{array}$ \\
\hline \multicolumn{5}{|l|}{ Antidepressants } \\
\hline Non-selective monoamine inhibitors & $35(60.3)$ & $26(36.7)$ & $31(22.2)$ & $24(15.2)$ \\
\hline Amitriptyline & $17(29.3)$ & $12(17.0)$ & $14(10.0)$ & $12(7.6)$ \\
\hline Amitriptyline + chlordiazepoxide & $4(6.9)$ & $6(8.5)$ & $5(3.6)$ & 0.0 \\
\hline Clomipramine & $2(3.4)$ & $3(4.2)$ & $6(4.3)$ & $3(1.9)$ \\
\hline Imipramine & $9(15.5)$ & $4(5.6)$ & $1(0.7)$ & $4(2.5)$ \\
\hline Nortriptyline & $3(5.2)$ & $1(1.4)$ & $5(3.6)$ & $5(3.2)$ \\
\hline Selective Serotonin Reuptake Inhibitors & $17(29.31)$ & $32(45.1)$ & $87(62.7)$ & $106(67.6)$ \\
\hline Citalopram & $1(1.7)$ & 0.0 & $3(2.2)$ & $10(6.4)$ \\
\hline Escitalopram & 0.0 & 0.0 & $4(2.9)$ & $12(7.6)$ \\
\hline Fluoxetine & $10(17.2)$ & $17(24.0)$ & $51(36.7)$ & $47(30.0)$ \\
\hline Paroxetine & $3(5.2)$ & $11(15.5)$ & $13(9.4)$ & $16(10.2)$ \\
\hline Sertraline & $3(5.2)$ & $4(5.6)$ & $16(11.5)$ & $21(13.4)$ \\
\hline Others & $3(5.1)$ & $3(4.2)$ & $16(11.5)$ & $22(13.9)$ \\
\hline Bupropion & 0.0 & $3(4.2)$ & $4(2.9)$ & $3(1.9)$ \\
\hline Desvenlafaxine & 0.0 & 0.0 & 0.0 & $1(0.6)$ \\
\hline Duloxetine & 0.0 & 0.0 & $2(1.4)$ & $6(3.8)$ \\
\hline Mirtazapine & $1(1.7)$ & 0.0 & $1(0.7)$ & 0.0 \\
\hline Trazodone & $1(1.7)$ & 0.0 & $4(2.9)$ & $4(2.5)$ \\
\hline Venlafaxine & $1(1.7)$ & 0.0 & $5(3.6)$ & $8(5.1)$ \\
\hline Only the class declared & $4(6.9)$ & $10(14.1)$ & $7(5.0)$ & $6(3.8)$ \\
\hline \multicolumn{5}{|l|}{ Benzodiazepines } \\
\hline Alprazolam & $8(4.2)$ & $24(12.4)$ & $28(12.2)$ & $21(10.5)$ \\
\hline Bromazepam & $83(43.7)$ & $66(34.0)$ & $43(18.8)$ & $24(12.0)$ \\
\hline Clobazam & $2(1.0)$ & 0.0 & $2(0.9)$ & $3(1.5)$ \\
\hline Clonazepam & $9(4.7)$ & $21(10.8)$ & $82(36.0)$ & $101(50.5)$ \\
\hline Clorazepate dipotassium & $1(0.5)$ & 0.0 & 0.0 & 0.0 \\
\hline Cloxazolam & $16(8.4)$ & $23(11.9)$ & $15(6.5)$ & $15(7.5)$ \\
\hline Diazepam & $41(21.6)$ & 38 (19.6) & $44(19.2)$ & $24(12.0)$ \\
\hline Flunitrazepam & 0.0 & $10(0.5)$ & $5(2.2)$ & $2(1.0)$ \\
\hline Flurazepam & $3(1.6)$ & $2(1.0)$ & $1(0.4)$ & $2(1.0)$ \\
\hline Lorazepam & $4(2.1)$ & $6(3.1)$ & $2(0.9)$ & $2(1.0)$ \\
\hline Midazolam & $3(1.6)$ & $4(2.1)$ & $3(1.3)$ & $2(1.0)$ \\
\hline Nitrazepam & 0.0 & $1(0.5)$ & 0.0 & 0.0 \\
\hline Only the class declared & $23(12.1)$ & $13(6.7)$ & $10(4.4)$ & $8(4.0)$ \\
\hline
\end{tabular}

One individual may be in more than one drug pharmacological class.

twice as high as that observed for married individuals. There was an increase in the incidence of BDZ use as the participants' perception of their health status worsened. There was a $34 \%$ higher incidence of BDZ use among those with a GHQ-12 score compatible with CMD, as well as a direct dose-response pattern for the waking up during the sleep cycle variable (Table 4).

\section{DISCUSSION}

In the study population, an increase in both AD and BDZ use was observed between 1999 and 2012, although this increase has been proportionally higher for the first drug (Figure 1). This pattern is consistent with what has been observed in other countries, having as probable causes the introduction of new AD pharmacological classes ${ }^{1}$, the reduced side-effects of the most recent $\mathrm{AD}^{1,2}$, and an expansion in the spectrum of $\mathrm{AD}$ indications ${ }^{3,18-20}$. 
Table 3. Cumulative incidence over 8 years (1999-2007) of antidepressant and benzodiazepine use stratified according to sociodemographic variables and self-reported health condition. Pró-Saúde Study, Brazil.

\begin{tabular}{|c|c|c|c|c|}
\hline & $\begin{array}{l}\text { Incidence of AD (\%) } \\
(95 \% \mathrm{Cl})\end{array}$ & p-value & $\begin{array}{c}\text { Incidence of BDZ (\%) } \\
(95 \% \mathrm{CI})\end{array}$ & p-value \\
\hline Total & $4.9(4.2-5.7)$ & & $8.3(7.3-9.3)$ & \\
\hline \multicolumn{5}{|l|}{ Sex } \\
\hline Men & $2.1(1.3-2.9)$ & \multirow{2}{*}{$<0.001$} & $5.3(4.1-6.6)$ & \multirow{2}{*}{$<0.001$} \\
\hline Women & $7.2(6.0-8.4)$ & & $10.6(9.1-12.2)$ & \\
\hline \multicolumn{5}{|l|}{ Age } \\
\hline $22-34$ & $4.9(3.4-6.4)$ & \multirow{4}{*}{0.20} & $6.0(4.4-7.7)$ & \multirow{4}{*}{$<0.001$} \\
\hline $35-44$ & $5.0(3.9-6.2)$ & & $8.2(6.7-9.7)$ & \\
\hline $45-54$ & $5.7(3.9-7.5)$ & & $10.0(7.6-12.3)$ & \\
\hline 55 or more & $1.7(0.0-3.7)$ & & $13.3(8.4-18.7)$ & \\
\hline \multicolumn{5}{|l|}{ Marital status } \\
\hline Married or living together & $4.2(3.3-5.1)$ & \multirow{4}{*}{0.08} & $7.2(6.2-8.4)$ & \multirow{4}{*}{$<0.001$} \\
\hline Separated or divorced & $6.8(4.5-9.2)$ & & $10.8(7.8-13.8)$ & \\
\hline Widower & $7.4(1.7-13.1)$ & & $22.2(13.1-31.3)$ & \\
\hline Single & $5.5(3.7-7.3)$ & & $8.2(6.0-10.5)$ & \\
\hline \multicolumn{5}{|l|}{ Race/color } \\
\hline Black & $4.1(2.3-5.9)$ & \multirow{5}{*}{0.27} & $11.3(8.4-14.2)$ & \multirow{5}{*}{0.02} \\
\hline Brown & $4.2(2.9-5.4)$ & & $7.2(5.5-8.8)$ & \\
\hline White & $5.9(4.7-7.1)$ & & $8.0(6.6-9.4)$ & \\
\hline Asian & $3.8(0.0-8.9)$ & & $7.8(0.4-15.3)$ & \\
\hline Indigenous & $3.1(0.0-9.2)$ & & $18.7(5.0-32.5)$ & \\
\hline \multicolumn{5}{|l|}{ Education level } \\
\hline Some elementary school & $2.8(0.6-5.1)$ & \multirow{7}{*}{0.03} & $9.9(5.8-14.0)$ & \multirow{7}{*}{0.50} \\
\hline Elementary school & $2.5(0.5-4.6)$ & & $6.5(3.3-9.7)$ & \\
\hline Some high school & $2.8(0.9-4.7)$ & & $8.1(4.8-11.4)$ & \\
\hline High school & $4.5(2.9-6.0)$ & & $9.0(6.9-11.2)$ & \\
\hline Some college & $6.7(4.3-9.1)$ & & $7.4(4.8-9.9)$ & \\
\hline College & $6.1(4.5-7.8)$ & & $7.3(5.5-9.1)$ & \\
\hline Graduate & $6.2(3.5-8.8)$ & & $10.3(7.0-13.7)$ & \\
\hline \multicolumn{5}{|l|}{ Workplace } \\
\hline UERJ Campus ${ }^{1}$ & $4.1(3.1-5.1)$ & \multirow{2}{*}{0.04} & $6.9(5.6-8.2)$ & \multirow{2}{*}{0.01} \\
\hline HUPE Hospital $^{2}$ & $5.7(4.6-6.9)$ & & $9.6(8.1-11.1)$ & \\
\hline \multicolumn{5}{|c|}{ Income equivalence (minimum wages) } \\
\hline Up to 3 & $3.5(1.3-5.8)$ & \multirow{3}{*}{0.45} & $8.7(5.1-12.3)$ & \multirow{3}{*}{0.96} \\
\hline 3 to 6 & $4.8(3.3-6.3)$ & & $8.1(6.2-10.1)$ & \\
\hline$>6$ & $5.3(4.3-6.3)$ & & $8.2(7.0-9.5)$ & \\
\hline \multicolumn{5}{|l|}{ Health insurance users } \\
\hline Yes & $5.6(4.5-6.7)$ & \multirow{2}{*}{0.10} & $8.7(7.4-10.1)$ & 032 \\
\hline No & $4.2(3.2-5.3)$ & & $7.7(6.3-9.2)$ & 0.02 \\
\hline Alcohol consumption & & & & \\
\hline Yes & $4.2(3.2-5.2)$ & 70 & $7.3(6.0-8.6)$ & 007 \\
\hline No & $5.7(4.4-6.9)$ & 0.07 & $9.2(7.6-10.8)$ & 0.07 \\
\hline Frequency of alcohol consun & (eeks) & & & \\
\hline Every day & $1.7(0.0-5.0)$ & & $3.6(0.0-8.6)$ & \\
\hline 10 to 13 days & $5.3(0.0-11.3)$ & & $9.2(1.4-17.0)$ & \\
\hline 6 to 9 days & $7.2(2.6-11.7)$ & 0.41 & $8.1(3.3-13.0)$ & 0.67 \\
\hline 2 to 5 days & $3.9(2.6-5.2)$ & & $6.9(5.2-8.7)$ & \\
\hline 1 single day & $4.2(2.5-6.0)$ & & $8.3(5.9-10.7)$ & \\
\hline
\end{tabular}


Table 3. Cumulative incidence over 8 years (1999-2007) of antidepressant and benzodiazepine use stratified according to sociodemographic variables and self-reported health condition. Pró-Saúde Study, Brazil. (Continuation)

\begin{tabular}{|c|c|c|c|c|}
\hline & $\begin{array}{c}\text { Incidence of AD (\%) } \\
(95 \% \mathrm{CI})\end{array}$ & p-value & $\begin{array}{c}\text { Incidence of BDZ (\%) } \\
(95 \% \mathrm{CI})\end{array}$ & p-value \\
\hline \multicolumn{5}{|c|}{ Amount of alcohol consumed per day (2 weeks) } \\
\hline 1 standard unit & $4.6(2.7-6.5)$ & \multirow{5}{*}{0.98} & $7.2(4.8-9.5)$ & \multirow{5}{*}{0.009} \\
\hline 2 to 4 standard unit & $4.1(2.6-5.6)$ & & $5.8(4.0-7.6)$ & \\
\hline 5 to 7 standard unit & $4.1(1.6-6.6)$ & & $13.0(8.7-17.3)$ & \\
\hline 8 a 10 standard unit & $3.8(0.1-7.4)$ & & $7.9(2.6-13.2)$ & \\
\hline More than 10 standard unit & $5.0(0.7-9.4)$ & & $6.5(1.4-11.6)$ & \\
\hline \multicolumn{5}{|l|}{ Health condition } \\
\hline Very good & $3.6(2.3-4.8)$ & \multirow{4}{*}{$<0.001$} & $5.1(3.6-6.5)$ & \multirow{4}{*}{$<0.001$} \\
\hline Good & $4.7(3.7-5.7)$ & & $7.9(6.6-9.2)$ & \\
\hline Regular & $8.0(5.6-10.5)$ & & $14.2(11.1-17.5)$ & \\
\hline Bad & $8.3(0.0-17.5)$ & & $22.8(8.7-37.0)$ & \\
\hline \multicolumn{5}{|l|}{ Current health condition } \\
\hline Better than 12 months ago & $5.1(3.3-6.9)$ & \multirow{3}{*}{0.004} & $12.1(9.4-14.9)$ & \multirow{3}{*}{$<0.001$} \\
\hline Same as 12 months ago & $4.3(3.5-5.2)$ & & $6.7(5.6-7.8)$ & \\
\hline Worse than 12 months ago & $8.4(5.6-11.2)$ & & $11.5(8.1-14.9)$ & \\
\hline \multicolumn{5}{|l|}{ GHQ-12 (Positive $\geq 3$ ) } \\
\hline Negative & $3.7(2.9-4.5)$ & \multirow{2}{*}{$<0.001$} & $6.3(5.3-7.4)$ & \multirow{2}{*}{$<0.001$} \\
\hline Positive & $8.0(6.2-9.7)$ & & $12.7(10.5-15.0)$ & \\
\hline \multicolumn{5}{|l|}{ Difficulty falling asleep } \\
\hline Always & $10.6(5.3-15.9)$ & \multirow{5}{*}{$<0.001$} & $21.7(13.8-29.6)$ & \multirow{5}{*}{$<0.001$} \\
\hline Almost Always & $4.6(1.8-7.3)$ & & $21.8(16.1-27.5)$ & \\
\hline Sometimes & $6.2(4.4-8.0)$ & & $9.4(7.2-11.2)$ & \\
\hline Rarely & $4.6(3.0-6.2)$ & & $6.9(5.0-8.9)$ & \\
\hline Never & $3.9(2.8-5.0)$ & & $5.0(3.8-6.2)$ & \\
\hline \multicolumn{5}{|l|}{ Wake up during sleep cycle } \\
\hline Always & $11.5(5.4-17.7)$ & \multirow{5}{*}{$<0.001$} & $19.5(11.1-27.9)$ & \multirow{5}{*}{$<0.001$} \\
\hline Almost Always & $3.4(1.1-5.7)$ & & $19.2(14.0-24.4)$ & \\
\hline Sometimes & $7.5(5.5-9.5)$ & & $9.9(7.6-12.3)$ & \\
\hline Rarely & $3.8(2.4-5.3)$ & & $7.5(5.5-9.5)$ & \\
\hline Never & $4.0(2.9-5.0)$ & & $5.1(3.5-6.4)$ & \\
\hline \multicolumn{5}{|l|}{ Tiredness for no apparent reason } \\
\hline Always & $13.9(8.0-19.7)$ & \multirow{5}{*}{$<0.001$} & $20.0(12.8-27.2)$ & \multirow{5}{*}{$<0.001$} \\
\hline Almost Always & $9.0(5.8-12.2)$ & & $17.0(12.7-21.3)$ & \\
\hline Sometimes & $6.2(4.6-7.9)$ & & $9.8(7.7-11.8)$ & \\
\hline Rarely & $2.8(14.5-4.2)$ & & $4.8(3.0-6.6)$ & \\
\hline Never & $3.0(2.0-4.0)$ & & $5.2(3.9-6.4)$ & \\
\hline
\end{tabular}

'Universidade Estadual do Rio de Janeiro

${ }^{2}$ Hospital University Pedro Ernesto

This result partially confirms our initial hypothesis, as there was an increase in the use of $\mathrm{AD}$, but not accompanied by a decrease in the BDZ use, but by more subtle increases in prevalence. That is, there was no substitution of BDZ by AD, but a possible association of both pharmacological classes to treat mental health disorders in some individuals ${ }^{21}$.

Paulose-Ram et al. ${ }^{22}$ also reported an increase in the prevalence of AD $(2.5 \%, 1988-1994$ versus 8.1\% 1999-2002) and BDZ use (3.5\%, 1988-1994 versus 3.8\%, 1999-2002) in the United States. However, a 1994 UK study found a reduction in the use of BDZ over time ${ }^{23}$. This reduced use of BDZ was also reported by other authors?.

A characteristic of the SSRI therapeutic approach is the recommendation of continuous and long-term treatment that may continue for up to 6 months or more after the symptoms 
Table 4. Poisson regression models for cumulative incidence of the antidepressants and benzodiazepines use. Pró-Saúde Study, Brazil.

\begin{tabular}{|c|c|c|c|c|}
\hline \multirow{2}{*}{ Variable } & \multicolumn{2}{|c|}{ Antidepressants } & \multicolumn{2}{|c|}{ Benzodiazepines } \\
\hline & $\mathrm{RR}(95 \% \mathrm{CI})$ & p-value & $\mathrm{RR}(95 \% \mathrm{CI})$ & p-value \\
\hline \multicolumn{5}{|l|}{ Sex } \\
\hline Men & 1 & \multirow{2}{*}{$<0.001$} & 1 & \multirow{2}{*}{0.001} \\
\hline Women & $2.66(1.75-4.04)$ & & $1.58(1.3-2.1)$ & \\
\hline \multicolumn{5}{|l|}{ Age } \\
\hline $22-34$ & 1 & & $\mathrm{NI}$ & $\mathrm{NI}$ \\
\hline $35-44$ & $1.15(0.78-1.70)$ & 0.47 & $\mathrm{NI}$ & $\mathrm{NI}$ \\
\hline $45-54$ & $1.54(1.0-2.44)$ & 0.062 & $\mathrm{NI}$ & $\mathrm{NI}$ \\
\hline 55 or more & $0.53(0.16-1.77)$ & 0.305 & NI & $\mathrm{NI}$ \\
\hline \multicolumn{5}{|l|}{ Marital status } \\
\hline Married or living together & $\mathrm{NI}$ & $\mathrm{NI}$ & 1 & \\
\hline Separated or divorced & $\mathrm{NI}$ & $\mathrm{NI}$ & $1.20(0.86-1.68)$ & 0.28 \\
\hline Widower & $\mathrm{NI}$ & $\mathrm{NI}$ & $2.15(1.34-3.47)$ & 0.002 \\
\hline Single & $\mathrm{NI}$ & $\mathrm{NI}$ & $1.03(0.82-1.55)$ & 0.46 \\
\hline \multicolumn{5}{|l|}{ Race/Color } \\
\hline Brown & $\mathrm{NI}$ & $\mathrm{NI}$ & 1 & \\
\hline Black & $\mathrm{NI}$ & $\mathrm{NI}$ & $1.47(1.03-2.09)$ & 0.03 \\
\hline White & $\mathrm{NI}$ & $\mathrm{NI}$ & $1.26(0.93-1.71)$ & 0.14 \\
\hline Asian & $\mathrm{NI}$ & $\mathrm{NI}$ & $1.25(0.47-3.27)$ & 0.65 \\
\hline Indigenous & $\mathrm{NI}$ & $\mathrm{NI}$ & $3.15(1.42-6.97)$ & 0.005 \\
\hline \multicolumn{5}{|l|}{ Education level } \\
\hline Some elementary school & 1 & & $\mathrm{NI}$ & $\mathrm{NI}$ \\
\hline Elementary school & $0.99(0.32-3.03)$ & 0.98 & $\mathrm{NI}$ & $\mathrm{NI}$ \\
\hline Some high school & $0.99(0.34-2.84)$ & 0.98 & $\mathrm{NI}$ & $\mathrm{NI}$ \\
\hline High school & $1.7(0.69-4.18)$ & 0.25 & $\mathrm{NI}$ & $\mathrm{NI}$ \\
\hline Some college/ graduate & $2.35(0.98-5.62)$ & 0.05 & $\mathrm{NI}$ & $\mathrm{NI}$ \\
\hline \multicolumn{5}{|l|}{ Health condition } \\
\hline Very good & $\mathrm{NI}$ & $\mathrm{NI}$ & 1 & \\
\hline Good & $\mathrm{NI}$ & $\mathrm{NI}$ & $1.4(0.98-2.00)$ & 0.06 \\
\hline Regular/Bad & $\mathrm{NI}$ & $\mathrm{NI}$ & $1.81(1.2-2.72)$ & 0.005 \\
\hline \multicolumn{5}{|l|}{ GHQ-12 (Positive $\geq 3$ ) } \\
\hline Negative & 1 & \multirow{2}{*}{0.08} & 1 & \\
\hline Positive & $1.34(0.96-1.88)$ & & $1.34(1.05-1.79)$ & 0.03 \\
\hline \multicolumn{5}{|l|}{ Wake up during sleep cycle } \\
\hline Never & $\mathrm{NI}$ & $\mathrm{NI}$ & 1 & \\
\hline Rarely & $\mathrm{NI}$ & $\mathrm{NI}$ & $1.23(0.85-1.77)$ & 0.27 \\
\hline Sometimes & $\mathrm{NI}$ & $\mathrm{NI}$ & $1.45(1.01-2.07)$ & 0.04 \\
\hline Almost Always/Always & $\mathrm{NI}$ & $\mathrm{NI}$ & $2.71(1.86-3.94)$ & $<0.001$ \\
\hline \multicolumn{5}{|c|}{ Tiredness for no apparent reason } \\
\hline Never & 1 & & $\mathrm{NI}$ & $\mathrm{NI}$ \\
\hline Rarely & $0.84(0.47-1.50)$ & 0.56 & $\mathrm{NI}$ & $\mathrm{NI}$ \\
\hline Sometimes & $1.73(1.11-2.70)$ & 0.015 & $\mathrm{NI}$ & $\mathrm{NI}$ \\
\hline Almost Always & $2.02(1.20-3.42)$ & 0.008 & $\mathrm{NI}$ & $\mathrm{NI}$ \\
\hline Always & $3.2(1.82-5.66)$ & $<0.001$ & $\mathrm{NI}$ & $\mathrm{NI}$ \\
\hline
\end{tabular}

$\mathrm{NI}$ - Not included in the final regression model since it presented a $\mathrm{p}$-value $>0.10$.

disappear. It is possible the use of $\mathrm{AD}$ in our sample was continuous, while for $\mathrm{BDZ}$ it was more short-term and episodic (to deal with anxiety and insomnia episodes).

In this study, SSRI were the most used AD; the proportion of their users nearly doubled from 1999 to 2012, agreeing with many studies ${ }^{14,24,25}$. 
With the increase in SSRI use, there was a decrease in the use of other antidepressants, especially the TCA (mostly amitriptyline). The proportion of TCA users among AD users decreased by 75\% between 1999 and 2012. This pattern agreed with other authors. In the US a reduction in the TCA use was observed, from $47 \%$ in 1987 to $2.1 \%$ by $2001^{1}$. In Spain, in 2001-02, the frequency of SSRI use was 59.5\%, and of TCA use was $25.5 \%$ among AD users $^{5}$. In the US, an increase in the prevalence of SSRI use was also observed; in 1999, the prevalence of SSRI use was 4.3\%, increasing to $8.5 \%$ in $2012^{25}$. In São Paulo, the prevalence of SSRI use was $2.17 \%$, and of TCA was $1.26 \%$ in $2005-2007^{11}$, while in Chile the prevalence of SSRI use was $2.0 \%$ and TCA use was $1.8 \%$ in $1996-98^{4}$.

When examining the use of both psychotropic medicines, the incidence of BDZ use between 1999 and 2007 was twice as high as that for AD. In a longitudinal study conducted in France with less follow-up time than this study (1996-2001), it was found 2.3\% of new AD users, $2.8 \%$ anxiolytics, and $2.3 \%$ hypnotics ${ }^{26}$.

In this study, the first time an individual used AD or BDZ was not assessed, but the use that started (or restarted) during follow-up among those who did not use psychotropic medication at baseline was assessed. Since the assessment of incidence excluded AD and BDZ users at baseline, there may have been a higher chance of detecting the beginning or the return to BDZ use (medication that has a more cyclic or episodic use) than the beginning or return to $\mathrm{AD}$ use (a medication that tends to have a more prolonged and continuous use). This fact may have contributed to the higher increase in the found AD use compared with BDZ use in our follow-up.

Being a BDZ user at baseline increased the risk of the individual of starting AD use. The data are not surprising, given the evidence that primary care initiates the treatment of some mental disorders with BDZ before referring the individuals to specialists ${ }^{3}$. The literature reports that the use of $\mathrm{BDZ}$ to treat depression is common and that this use may continue even after the introduction of $\mathrm{AD}^{3}$. The use of $\mathrm{AD}$ was also found by Takeshima et al. ${ }^{27}$ to be a predictor not only for the initial use of BDZ but also as a predictor of prolonged used.

Cumulative incidence in the period from 1999 to 2006-07 was higher among women, both for $\mathrm{AD}$ and $\mathrm{BDZ}$ as others have observed ${ }^{24,26}$. Anxiety disorders and depression have been consistently described as being more frequent among women ${ }^{7,14}$, who also have a higher tendency to seek medical care ${ }^{7}$; besides, new indications for $\mathrm{AD}$ use encompasses ailments that are specifically or primarily suffered by women, such as premenstrual syndrome, premenstrual dysphoric disorder, eating disorders, fibromyalgia, and headaches ${ }^{18,19,20,24}$.

There was an increase in the incidence of BDZ with age. However, in the AD case, the very oldest age group presented a significant reduction in incidence. This decrease in our sample may be due to a healthy worker effect ${ }^{28}$; the small size of this age group may have also affected the results.

Increased education level was associated with the incidence of $\mathrm{AD}$ use, but not with BDZ use. Some hypotheses may explain this result. Individuals with a greater education level may describe their condition more clearly, leading to a more adequate treatment. These individuals may seek help from a psychiatrist rather than a general practitioner ${ }^{29}$; furthermore, in general they have higher incomes, allowing them to afford the AD cost, usually higher than the BDZ cost.

The incidence of BDZ use was higher among individuals that classified their health status as poor, consistently with the fact that chronic health conditions, both physical and mental, increase the search for professional help and the prescription of psychotropic medicine ${ }^{26,30}$. In the bivariate analysis, AD was also shown to be associated with the worst health perception. The fact that this association was not maintained in the multivariate analysis may be due to less statistical power in the analysis of these medicines, due to lower incidence when compared with BDZ. The incidence of BDZ use was also higher among people who reported having trouble falling and remaining asleep. This result is expected considering that BDZ is broadly prescribed worldwide for such conditions ${ }^{27}$. However, complaints of tiredness in the baseline were associated with higher $\mathrm{AD}$ use, which can be explained because of this complaint association with depressive disorders. 
This study reported the cumulative incidence of $\mathrm{AD}$ and $\mathrm{BDZ}$ use over a 13-year period, results that are unprecedented. Among the study limitations, the fact of not covering the medication use for the entire follow-up may have non-differentially misclassified some participants, therefore producing an underestimation of the incidence estimates. On the other hand, this restriction may have minimized the memory bias.

Contrary to our hypothesis and to the findings reported by other studies, this analysis did not corroborate the previous impression that the use of BDZ was being gradually replaced by the use of $\mathrm{AD}$ for many individuals. These results did not show an increase in the incidence of $\mathrm{AD}$ use followed by a decrease in the incidence of $\mathrm{BDZ}$ use. The reasons for such phenomenon are not clear. It is likely that AD is being added to the use of BDZ, and not just substituting the latter in the study population. It is imperative that $\mathrm{AD}$ and $\mathrm{BDZ}$ prescriptions are in agreement with the current treatment guidelines, especially considering the rational use of psychotropic medications.

\section{REFERENCES}

1. Stafford RS, MacDonald EA, Finkelstein SN. National patterns of medication treatment for depression, 1987 to 2001. Prim Care Companion J Clin Psychiatry. 2001;3(6):232-5. https://doi.org/10.4088/pcc.v03n0611

2. Subramaniam M, He VY, Vaingankar JA, Abdin E, Chong SA. Prevalence of and factors related to the use of antidepressants and benzodiazepines: results from the Singapore Mental Health Study. BMC Psychiatry. 2013;13:231. https://doi.org/10.1186/1471-244X-13-231

3. Baldwin DS, Allgulander C, Bandelow B, Ferre F, Pallanti S. An international survey of reported prescribing practice in the treatment of patients with generalised anxiety disorder. World J Biol Psychiatry. 2012;13(7):510-6. https://doi.org/10.3109/15622975.2011.624548

4. Rojas G, Fritsch R, Gaete J, González I, Araya R. Use of psychotropic medication in Santiago, Chile. J Ment Health. 2005;14(4):407-14. https://doi.org/10.1080/09638230500195221

5. Codony M, Alonso J, Almansa J, Vilagut G, Domingo A, Pinto-Meza A, et al. Uso de fármacos psicotrópicos en España: resultados del estudio ESEMeD-España. Actas Esp Psiquiatr. 2007;35 Supl 2:29-36.

6. Bruffaerts R, Bonnewyn A, Oyen H, Demarest S, Demyttenaere K. Consommation de médicaments psychotropes dans la population belge. Résultats de l'European Study of the Epidemiology of Mental Disorders (ESEMeD). Rev Med Liege. 2005;60(3):181-8.

7. Alonso J, Angermeyer MC, Bernert S, Bruffaerts R, Brugha TS, Bryson H, et al. Psychotropic drug utilization in Europe: results from the European Study of the Epidemiology of Mental Disorders (ESEMeD) Project. Acta Psychiatr Scand Suppl. 2004(420):55-64. https://doi.org/10.1111/j.1600-0047.2004.00331.x

8. Almeida LM, Coutinho ESF, Pepe VLE. Consumo de psicofármacos em uma região administrativa do Rio de Janeiro: a Ilha do Governador. Cad Saude Publica. 1994;10(1):5-16. https://doi.org/10.1590/S0102-311X1994000100002

9. Quintana MI, Andreoli SB, Moreira FG, Ribeiro WS, Feijo MM, Bressan RA, et al. Epidemiology of psychotropic drug use in Rio de Janeiro, Brazil: gaps in mental illness treatments. PLoS One. 2013;8(5):e62270. https://doi.org/10.1371/journal.pone.0062270

10. Mari JJ, Almeida-Filho N, Coutinho E, Andreoli SB, Miranda CT, Streiner D. The epidemiology of psychotropic use in the city of São Paulo. Psychol Med. 1993;23(2):467-74. https://doi.org/10.1017/s0033291700028555

11. Campanha AM, Siu ER, Milhorança IA, Viana MC, Wang Y-P, Andrade LH. Use of psychotropic medications in São Paulo Metropolitan Area, Brazil: pattern of healthcare provision to general population. Pharmacoepidemiol Drug Saf. 2015;24(11):1207-14. https://doi.org/10.1002/pds.3826

12. Quintana MI, Andreoli SB, Peluffo MP, Ribeiro WS, Feijo MM, Bressan RA, et al. Psychotropic drug use in São Paulo, Brazil: an epidemiological survey. PLoS One. 2015;10(8):e0135059. https://doi.org/10.1371/journal.pone.0135059

13. Lima MCP, Menezes PR, Carandina L, Cesar CLG, Barros MBA, Goldbaum M. Transtornos mentais comuns e uso de psicofármacos: impacto das condições socioeconômicas. Rev Saude Publica. 2008;42(4):717-23. https://doi.org/10.1590/S0034-89102008005000034 
14. Colman I, Croudace TJ, Wadsworth MEJ, Jones PB. Factors associated with antidepressant, anxiolytic and hypnotic use over 17 years in a national cohort. J Affect Disord. 2008;110(3):234-40. https://doi.org/10.1016/j.jad.2008.01.021

15. Faerstein E, Chor D, Lopes CS, Werneck GL. Estudo Pró-Saúde: características gerais e aspectos metodológicos. Rev Bras Epidemiol. 2005;8(4):454-66. https://doi.org/10.1590/S1415-790X2005000400014

16. Mari JJ, Williams P. Misclassification by psychiatric screening questionnaires. J Chronic Dis.1986;39(5):371-8. https://doi.org/10.1016/0021-9681(86)90123-2

17. WHO Collaborating Centre for Drug Statistics Methodology. Guidelines for ATC classification and DDD assignment. Oslo: Norwegian Institute of Public Health; 2019.

18. Steinberg EM, Cardoso GMP, Martinez PE, Rubinow DR, Schmidt PJ. Rapid response to fluoxetine in women with premenstrual dysphoric disorder. Depress Anxiety. 2012;29(6):531-40. https://doi.org/10.1002/da.21959

19. McQuay HJ, Tramér M, Nye BA, Carroll D, Wiffen PJ, Moore RA. A systematic review of antidepressants in neuropathic pain. Pain. 1996;68(2-3):217-27. https://doi.org/10.1016/S0304-3959(96)03140-5

20. Tomkins GE, Jackson JL, O'Malley PG, Balden E, Santoro JE. Treatment of chronic headache with antidepressants: a meta-analysis. Am J Med. 2001;111(1):54-63. https://doi.org/10.1016/s0002-9343(01)00762-8

21. Valenstein M, Taylor KK, Austin K, Kales HC, McCarthy JF, Blow FC. Benzodiazepine use among depressed patients treated in mental health settings. Am J Psychiatry. 2004;161(4):654-61. https://doi.org/10.1176/appi.ajp.161.4.654

22. Paulose-Ram R, Safran MA, Jonas BS, Gu Q, Orwig D. Trends in psychotropic medication use among U.S. adults. Pharmacoepidemiol Drug Saf. 2007;16(5):560-70. https://doi.org/10.1002/pds.1367

23. Ohayon MM, Caulet M, Priest RG, Guilleminault C. Psychotropic medication consumption patterns in the UK general population. J Clin Epidemiol. 1998;51(3):273-83. https://doi.org/10.1016/S0895-4356(97)00238-2

24. Noordam R, Aarts N, Verhamme KM, Sturkenboom MCM, Stricker BH, Visser LE. Prescription and indication trends of antidepressant drugs in the Netherlands between 1996 and 2012: a dynamic population-based study. Eur J Clin Pharmacol. 2015;71(3):369-75. https://doi.org/10.1007/s00228-014-1803-x

25. Kantor ED, Rehm CD, Haas JS, Chan AT, Giovannucci EL. Trends in prescription drug use among adults in the United States from 1999-2012. JAMA. 2015;314(17):1818-31. https://doi.org/10.1001/jama.2015.13766

26. Boeuf-Cazou O, Niezborala M, Marquie JC, Lapeyre-Mestre M. Factors associated with psychoactive drug initiation in a sample of workers in France: results of the VISAT cohort study. Pharmacoepidemiol Drug Saf. 2010;19(3):296-305. https://doi.org/10.1002/pds.1911

27. Takeshima N, Ogawa Y, Hayasaka Y, Furukawa TA. Continuation and discontinuation of benzodiazepine prescriptions: a cohort study based on a large claims database in Japan. Psychiatry Res. 2016;237:201-7. https://doi.org/10.1016/j.psychres.2016.01.040

28. Shah D. Healthy worker effect phenomenon. Indian J Occup Environ Med. 2009;13(2):77-9. https://doi.org/10.4103/0019-5278.55123

29. Halonen JI, Koskinen A, Kouvonen A, Varje P, Pirkola S, Väänänen A. Distinctive use of newer and older antidepressants in major geographical areas: a nationally representative register-based study. J Affect Disord. 2018;229:358-63. https://doi.org/10.1016/j.jad.2017.12.102

30. Salo P, Sivertsen B, Oksanen T, Sjösten N, Pentti J, Virtanen M, et al. Insomnia symptoms as a predictor of incident treatment for depression: prospective cohort study of 40,791 men and women. Sleep Med. 2012;13(3):278-84. https://doi.org/10.1016/j.sleep.2011.06.022

Authors' contributions: Study conception and planning and data analysis and interpretation, elaboration or revision of the manuscript, approval of the final version to be published, public responsibility for the content of the article: GCA, ESFC, EF.

Conflict of Interest: The authors declare no conflict of interest. 